\title{
Facial Recognition of Human in A Real Time Video Using PCA Algorithm
}

\author{
J Hemamalini $^{* 1}$, Sri Dhanya ${ }^{\# 2}$, Nikita Idiculla ${ }^{\# 3}$ \\ * Department of Information Technology, \\ Sathyabama University, Chennai -119, TamilNadu1 \\ ${ }^{1}$ hemamalini.it@sathyabamauniversity.ac.in \\ * Final Year Student, Department of Information Technology \\ Sathyabama University, Chennai-119, Tamilnadu. \\ ²Sridhanya009@gmail.com \\ ${ }^{3}$ niki.idiculla@gmail.com
}

\begin{abstract}
The human facial recognition has been attractive research area in computer vision. An existing facialrecognition system is based on still images, facing complex problem in discriminating foreground frombackground cluster without motion information. To overcome this problem the facial recognition invideo motion is projected. The execution work is based on haar cascading classification for facedetection and PCA (Principal Component Analysis) for Facial recognition. The database is trained with100 sample faces of 10 people with different poses, known as positive and negative example ofarbitrary image of the size 20 x20. The experimental results are compared with fisher face andLBP(Local binary pattern) but PCA provides $92.4 \%$ accuracy.
\end{abstract}

Keyword-Haar Cascading Classifier, PCA, Facial Recognition, Face Detection, Poses

\section{INTRODUCTION}

In the field of computer vision, one of the most important and curial area is the facial recognition. The aim of facial recognition is to dynamically analyze and recognize human face from a video. The human recognition is one of the most powerful tool, that the human can identify the unique feature from their faces. Thus the recognition of face in human through facial recognition has become a great deal during the last decades. The ability to detect and recognize human faces from videos helps to monitor the behaviour of objects. The several applications based on surveillance, which are used in ATM centers, Railway stations, companies and so on. These applications are mainly used to prevent criminal activities, and it also helps to monitor state and behaviour of human. In recent studies, many algorithms are being focused on face recognition, age calculation and facial emotion classifications. In this context, we implemented face recognition system based on PCA algorithm, it consist of Reading real time video from webcam, Face Detection, Face Extraction, Face alignment and finally recognition and representation is done by using Database. Some of the drawbacks in face detection system is to detect the face in which the person wears glass, hat and having moustache on his face. The recognition work based on capturing a real time video using webcam and clipped into many frames. Each frame is referred by Haar cascade trained classifier. The Haar feature based classifier is an effective object detection method to detect the face from frame. The detected face is taken for face estimation. In this context, the feature alignment of detected face is constructed through the ratios of the facial features for instance nose, eyes, lips, and so on. The alignment value of detected face is used for recognisation, by comparing the detected face with the database image. The database is enclosed with set of human face with details like name. The details of the human face are saved by the training set and can also be deleted. This system can detect multi-faces. However it can recognize only one face at a time.

\section{EXISTING SYSTEM}

An existing recognition system is based on still images, for recognition purpose the collection of still images are taken as input dataset. Each image is separately processed by eliminating background and foreground of an image. The designed descriptors for this facial recognition system are based on set of factors like relative positions, size and distance of faces for investigating human recognition 1. Some of the suitable algorithm for human face detection, such as Local Binary Pattern (LBP), Color Descriptor, Brightness Binary Feature (BBF), Integral Channel Features (ICF), Aggregate Channel Features (ACF), Locally Decorralated Channel Features (LDF), Informed Haar-like Features (IHF), Histograms of Oriented Gradients HOG, etc.,2,3,4. In current face detection algorithm provides less satisfactory for complex situations. For an example the view of face in different angle, illumination, occlusion, weather changes, facial appearance, shape, etc., at present the multi-view face detection is still quite challenging task [17]. Recognizing face from the video is difficult task, 
because detected face image is smaller than the frame size, for this the recognition needs a lot of improvement has to be made. For improvement various algorithms are used, which are Template Based approaches, Statistical approach, Model-based approaches, Wholistic approaches. The template Based approach is use an Adaptative Appearance Models. The Statistical approach of recognition algorithms are PCA(Principle component Analysis), ICA(Independent component analysis), LDA (Latent Dirichlet allocation),Discrete Cosine Transform, EP (Eigenspace), Kernel Method, Bayesian Framework, SVA (Singular value decomposition), 3-D Face Recognition, etc.

\section{SYSTEM ARCHITECTURE}

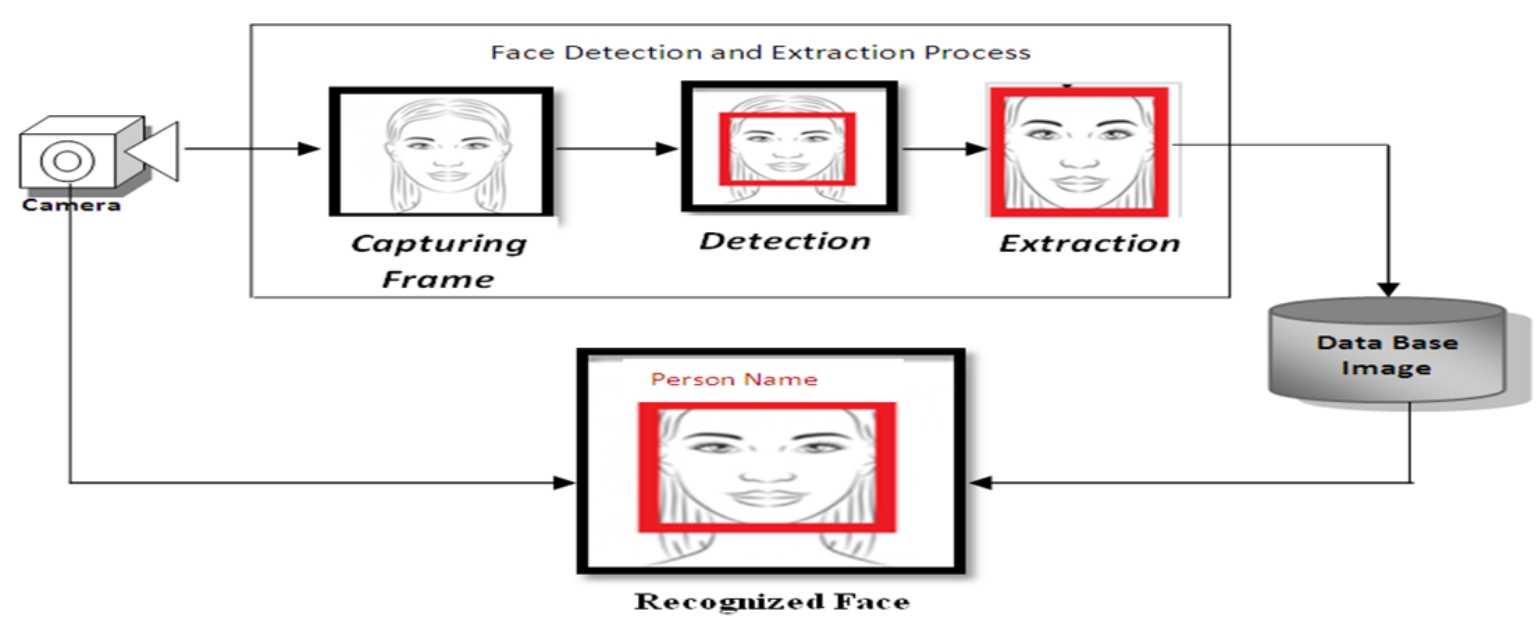

Fig 1. System Architecture

\section{CAPTURING VIDEO}

The first phase of facial recognition system is to acquire real time video by using webcam, as an input video stream is used for the face detection process. The accuracy of the video depends on the distance of object. The reading video is processed by capturing a frame from the video.

\section{FACE DETECTION}

The face detection is a technique in computer vision. It is used to identify the human face in a real time digital video, which refers psychological process of locating and identifying human face for various applications which are surveillance, authentication, etc., In this context, each frame of real time video is converted into gray level image, then the genetic algorithm of Haar-Cascade Classifier is used to locate the face in a frame. The classifier is trained with few sample faces known as positive examples, they are assigned with a particular size 20 X 20 and negative examples will act as the arbitrary images of the same assigned size. After the classifier is trained, it can by allot to the desired region in an input image. If the result of classifier is ' 1 ', mean that the selected region is depicting to show the face or else it results as ' 0 '. The design of the classifier is so efficient, that can be resized for better detection purpose. The detection region is processed by comparing the facial templates. The detection will depend on factors like distance, clarity, motion etc. Finally the detected face is highlighted using a rectangular box.

The key features of Haarcascade classifier are:

\section{Step 1: Calculate the Integral Image}

An Integral image is a process of calculating Summed Area Table (sum of values or pixel values). In this process every block is the summation of the previous blocks above it [2] as shown in the figure 2. Here the point of origin is the top left corner of the block and the previous blocks are those to the left of the as well as the above blocks. Its purpose is to allow quick computation of any area in an image with only four memory lookups and three addition or subtraction operations. These produces two equations, they are:

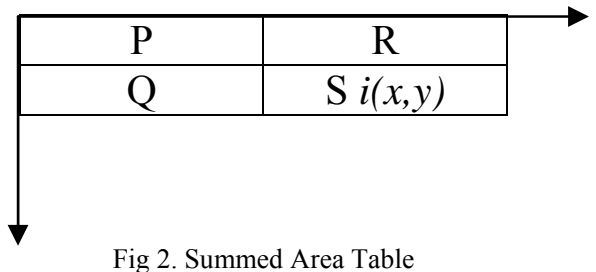


i ) Integral Image (I) of pixel at $x, y$

$$
\mathrm{I}=\operatorname{pixel}(\mathrm{S})+\operatorname{sum}(\mathrm{R})+\operatorname{sum}(\mathrm{S})-\operatorname{sum}(\mathrm{P})
$$

Where pixel is the total pixel of the particular block and sum() is the summation of pixels.

ii) Area (A) of a block at x, y (It works backwards)

Where sum() is the summation of pixels.

$$
A=\operatorname{sum}(S)-\operatorname{sum}(R)-\operatorname{sum}(S)+\operatorname{sum}(P)
$$

Example: Take an image with intensity of each pixel as shown in figure 3.

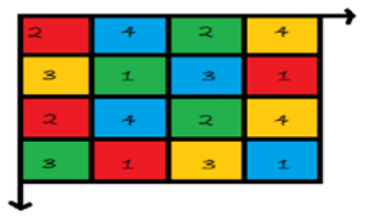

Fig3. Frame Intensity Value

Integral image of block $=$ summation of itself + summation of intensity of above block + summation of intensity of left block- intensity of the top left corner block.

Here, integral image of Red block $=2+0+0-0=2$, Integral image of Yellow block $=3+2+0-0=5$, Integral image of Blue block $=4+2+0-0=6$, Integral image of green block $=1+6+5-2=10$.

Similarly integral image of every block is done. The output is known as the integral summed area table. It will be as shown figure 4 .

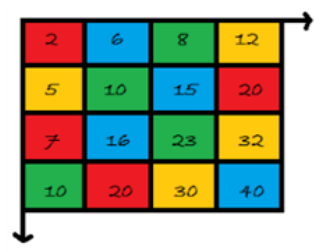

Fig 4. Summed Area Table

The integral image is obtained; this makes the calculation of any rectangular block in the image.

Eg: To find the area of a 2x 2 pixel block (Consider the block WXYZ) as shown in figure 5.

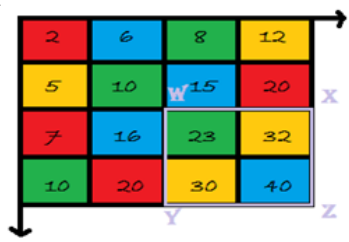

Fig5. Summed area table of $2 \times 2$ pixel block

Area $=\mathrm{Z}-\mathrm{Y}-\mathrm{X}+\mathrm{W}$

$=40-20-20+10=10$.

(From Eq.2)

Area of the original image

$=2+4+3+1=10$.

(From Eq.1)

\section{Step 2: Apply Haar-Features}

The Haar Feature is a digital features, it is used for recognition purpose. They are simple rectangular features that achieve random accuracy and are mainly used for their simplicity and fast computation time [2]. It is known as difference calculation. The difference between the entire whites block and the black blocks i.e, feature $=\operatorname{Sum}($ White $)$-Sum(Black).

There are numerous haar-like features available. Choose a subset of 5 more basic features. They are:

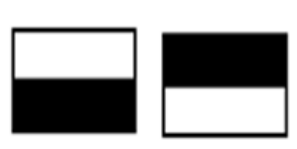

(i) Two edge features.

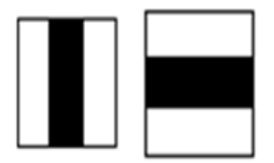

(ii) Two line features.

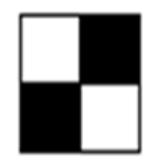

(iii) One four-rectangular features.

Fig 6.Haar Feature 
Based on the integral features, the edge features havesix memory lookups (two points at bottom, two points in middle and two points at the top).The line features have eight memory lookups(two points at the bottom, four points in the middle and two points at the top).The four-rectangular features have nine memory lookups (three points at bottom, two points in middle, one point at the centre and three points at the top). These variables are programmed with adjustable height and width dimensions for scaling[2].

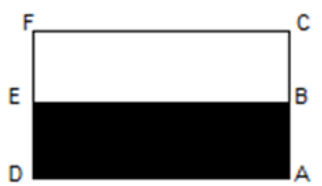

Two Rectangle $=\mathrm{A}-2 \mathrm{~B}+\mathrm{C}-\mathrm{D}+2 \mathrm{E}-\mathrm{F}$. (Two edge features)

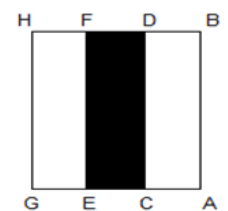

Three Rect $=\mathrm{A}-\mathrm{B}-2 \mathrm{C}+2 \mathrm{D}+2 \mathrm{E}-2 \mathrm{~F}-\mathrm{G}+\mathrm{H}$.

(Two line features)

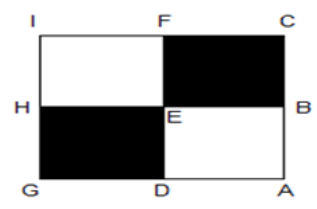

Four Rect $=\mathrm{A}-2 \mathrm{~B}+\mathrm{C}-2 \mathrm{D}+4 \mathrm{E}-2 \mathrm{~F}+\mathrm{H}-2 \mathrm{I}+\mathrm{J}$

(One four-rectangular features)

Fig 7. Haar Features

Step 3: Use Adaboost Learning Algorithm

It stands for adaptive boosting and is essentially the training algorithm that selects the best features out of the enormous over complete set of features available and creates a strong classifier. This algorithm is an aggressive approach which disregard the majority of features and results a fewer features. This provides a weak classifier (Wj)which consists of Features (Fj), Threshold ( $(\mathrm{Oj})$, Polarity (Pj).

$$
\mathrm{Wj}(\mathrm{x})= \begin{cases}1, i f \operatorname{Pj} F j(x)<P j \Theta j \\ 0, & \text { Otherwise }\end{cases}
$$

The positive images are only trained. Set the threshold value based on the average pixel intensities and standard deviation. This helps in faster implementation of code and require less data.

\section{Step 4:Apply Cascade Filter}

This process essentially discards all the negative sub-windows as earily as possible to reduce the over all computational time and focus more on possible positive windows. This is a binary tree created with all strong features from the adaboost method. This process allows possitive matches only to be revaluated at any point anything that registers its value as negative sub-window gets immediately rejected and exists. It reduces computation time spend on false windows by rejecting them. The threshold values can be adjusted to allow certain accuracy. Lower thresholds yeild higher detection rates but increase the occurance of false positives.

\section{TRAINING SET CREATION}

The extracted face of Haar-Cascade Classifier is dynamically stored in a database, which are used as training sets for recognition process. The training set contains details about the extracted face feature.

\section{FACE RECOGNITION}

Facial recognition is a biometric method, to identify an individual face by comparing live capture or digital image data with the database for that person. Facial recognition system is commonly used for security purposes but is increasingly being used in a variety of other applications. The facial recognition system is done by using Principal Component Analysis (PCA) algorithm. PCA is used for analyzing data (feature)and it is used to reduce the dimensionality of the feature. The major advantage of PCA is to reduce noise sensitivity, and increase efficiency. PCA facial recognition was performed by construction of Euclidean distance between feature vectors of detected face. PCA algorithm use covariance matrix and eigenvector for recognition. Eigenvector of the covariance matrix of the set of face image $\beta(\mathrm{a}, \mathrm{b})$, where an image with $\mathrm{N} \times \mathrm{N}$ pixel is considered a point in $\mathrm{N} 2$ dimensional space [7].

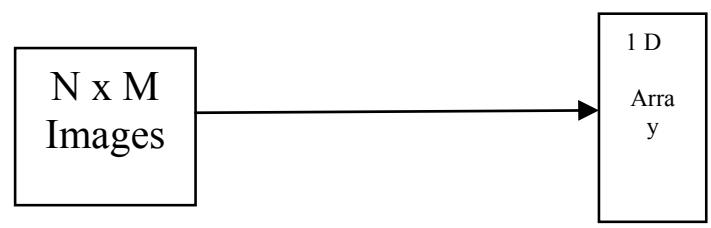

Fig 8. Conversations of $\mathrm{N} 2 \times \mathrm{M}$ to $\mathrm{MN} \times 1$ vector 
Step 1: Training process

Here the Training face are represented as $\mathrm{T} 1, \mathrm{~T} 2, \mathrm{~T} 3, \ldots \ldots \ldots . \mathrm{TM}$ with $\mathrm{m}$ images of size $\mathrm{N} \mathrm{x} \mathrm{N}$ are represented by vector size N2. Each face is represented by a variable $\beta 1, \beta 2, \beta 3$, placed into a training set $Z$. $Z$ contains $\{\beta 1, \beta 2, \beta 3$, ... $, \beta \mathrm{m}\}$

Step 2: Compute average face vector

To calculate average face vector $(\delta)$ use the formula,

Step 3: Covariance matrix $(\mathrm{C})$

$$
\delta=\frac{1}{M} \sum_{n-1}^{M} \beta \mathrm{n}
$$

Subtract average face vector from each face vector and stored it in a variable fIto calculate Covariance matrix (C).

$$
\begin{gathered}
£ \mathrm{i}=\beta 1-\delta(5) \\
\mathrm{C}=\mathrm{SS}^{\mathrm{T}}\left(\mathrm{N}^{2} \times \mathrm{N}^{2} \text { matrix }\right)
\end{gathered}
$$

Where $\mathrm{S}=[£ 1, £ 2, £ 3, \ldots \ldots \ldots, £ \mathrm{M}](\mathrm{N} 2 \times \mathrm{M}$ matrix $)$.

Step 4: Calculate the eigenvector and eigen values of the covariance matrix

The dimensionality of the covariance matrix is $\mathrm{N} 2 \times \mathrm{N} 2$. Here the total number of eigenvector will be greater than the total number of training set. For example: image of size $84 \times 84$ create the matrix size 7056 . Since the eigenvector of covariance matrix works on a converted vector form of image as it doesn't work on the image directly.

Consider the eigenvector yiofC $=\mathrm{SST}$

Consider the vectorriofC' $=$ STS $($ M x M matrix $)$

The relationship between yi and ri

By multiply S on both side

$$
\text { ST S ri }=\text { oiri }
$$

$$
\begin{array}{lc}
\text { C S ri }=\text { oiSri } & (\text { since } \mathrm{C}=\mathrm{SST}) \\
\text { C } y i=\sigma i S r i & \text { Whereyi }=\mathrm{S} \mathrm{ri}
\end{array}
$$$$
\text { ST Sri }=\sigma i S r i
$$

Thus $\mathrm{C}=$ SSTand $\mathrm{C}^{\prime}=$ STS have the same eigenvalues and their eigenvector are related as follows

$$
y i=S r i
$$

The final result of this derivation is that $\mathrm{S}$ ri is an eigenvector of $\mathrm{C}=\mathrm{SST}$. yi forms the eigenface of $\mathrm{M}$ eigenvector of $\mathrm{C}^{\prime}=$ STS

Step 5: Represent each face image a linear combination of all $\mathrm{K}$ eigenvector.

Each face from training set can be represented a weighted some of the $\mathrm{k}$ eigenface and the mean face because the removed average face vector must be added. If percentage of eigenvector is multiplied with the total eigenvector the percentage of resemblance of the original face can be obtained.

\section{RESULTS AND DISCUSSION}

The facial recognition system of human in a real-time video using PCA algorithm was implemented in C\#.Net. The result of PCA Algorithm is shown in the figure 9,10,11,12,13. Here sensitivity to variations in pose of face and illumination changes is still a challenging problem. The performance evaluation of the facial recognition system of identification rate (\%) as shown in the Table 1.

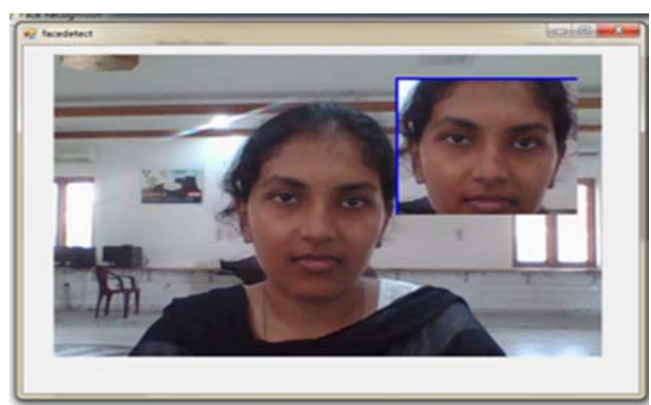

Fig 9. Detection of human face

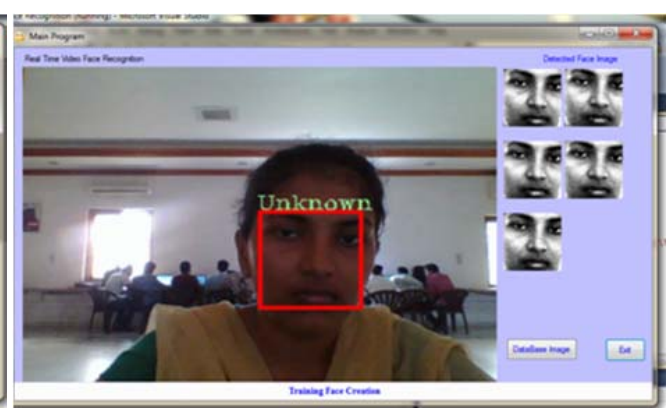

Fig 10. Detected Face 

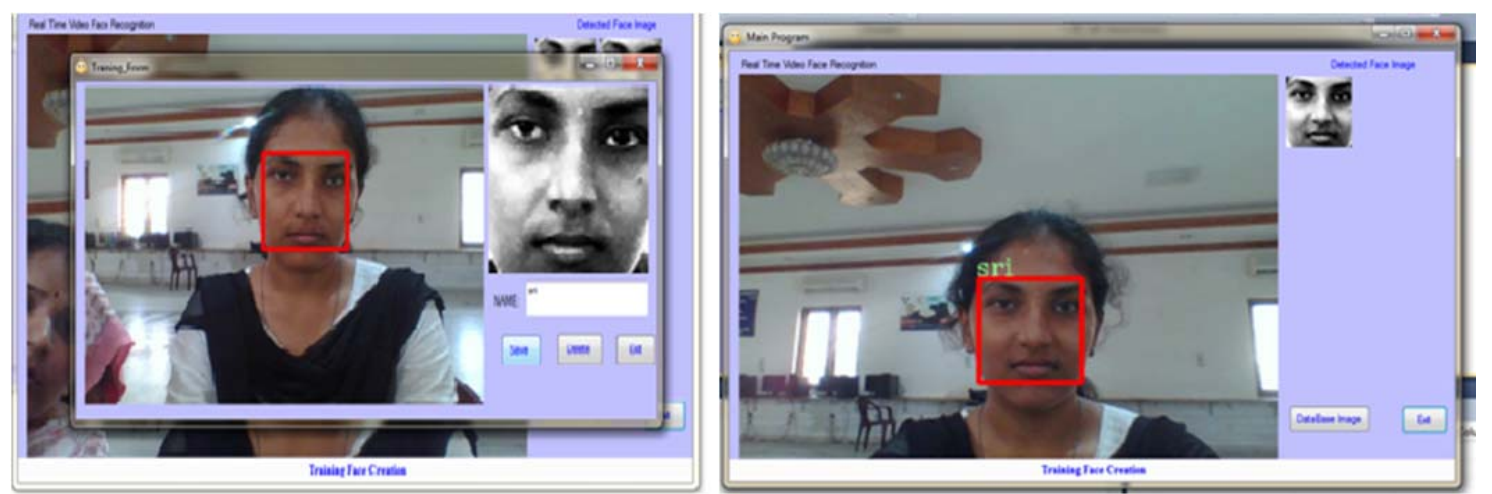

Fig11. Creation of Training SetFig 12. Recognizing Face by Matching with Database Image

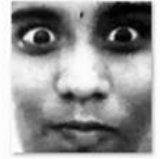

face_madhu_726 809554

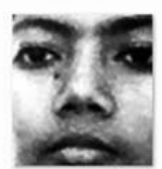

face_sneha_ 74502 7638

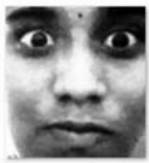

face_madhu_160 0576293

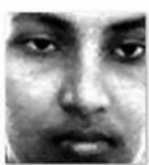

face_sri_-5

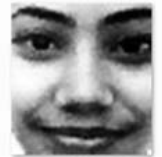

face nidhi 16293 82784

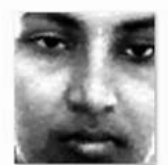

face_sri_16689762 33

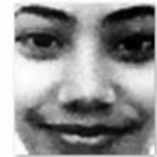

face nidhi 18865 05615

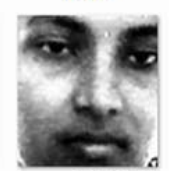

face_sri_16997129 35

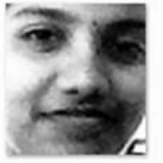

face_princy_511 75598

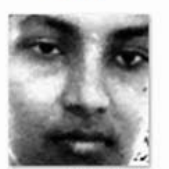

face_sri_19801754 96
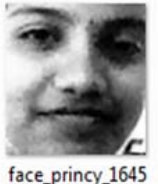
790352

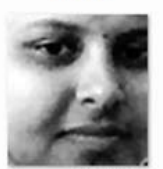

face_stefi_60315 938

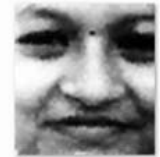

face salini 15472 09883

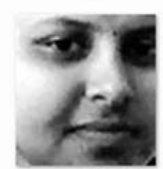

face_stefi_ 665916 082

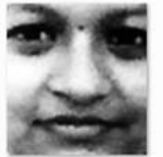

face_salini_19167

$$
29389
$$

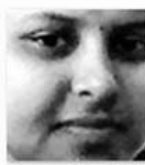

face_stefi_13938

8412

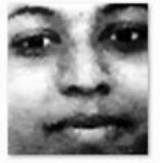

face_shruti_10266 12995

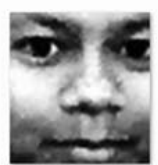

face_suhi_140904 9225

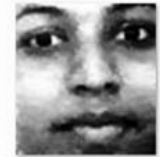

face_shruti_16745 28131

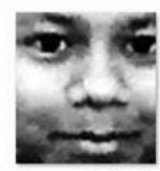

face_suhi_147286 7622

TABLE I

Performance evaluation of facial recognition system

\begin{tabular}{|l|l|c|c|}
\hline \multirow{4}{*}{ Algorithm } & & \multicolumn{2}{|c|}{ Identification Rate } \\
\cline { 3 - 4 } & & Front Face (\%) & Side face (\%) \\
\hline \multirow{4}{*}{ PCA } & Accuracy & 92.4 & 87.74 \\
\cline { 2 - 4 } & Error Rate & 19.8 & 13.4 \\
\cline { 2 - 4 } & Computation Time & 108 & 108 \\
\hline \multirow{4}{*}{ LBP } & Accuracy & 89.07 & 91.76 \\
\cline { 2 - 4 } & Error Rate & 8.7 & 7.3 \\
\cline { 2 - 4 } & Computation Time & 109 & 109 \\
\hline \multirow{3}{*}{ Fisher Face } & Error Rate & 70.75 & 91.56 \\
\cline { 2 - 4 } & Computation Time & 109 & 8.1 \\
\cline { 2 - 4 } & Accuracy & & 109 \\
\hline
\end{tabular}

The training set is created by assigning Authentication names to each detected face. The training set image is uploaded in the database for identification. The database contains 100 image, few of them are shown in figure 13. if any detected face match with the database image, it will display name at top the face else it will display unknown.

\section{CONCLUSION}

The human face is detected and recognized by using Haar Cascade Classifier algorithm and PCA algorithm. The database is trained with few sample faces known as positive example and negative example which will act as the arbitrary image of the same size $20 \times 20$. The database contains 100 images of 10 people with different poses. Some features where not detected, like the person wearing glass, hat and having moustache on his face. Advantage of PCA is to reduce the dimensionality of features by using covariance matrix and eigenvector. Here the human faces are recognized with the training set where the images are stored in the database. This system helps to detect multiple faces. Comparative study is done with LBP and Fisher face algorithm, the experimental result of PCA provides $92.4 \%$ accuracy. 


\section{ACKNOWLEDGMENT}

I would like to thank the management of Sathyabama University for their support in publishing this paper

\section{REFERENCES}

[1] GokhanTanisik, CemilZalluhoglu, NazliIkizler-Cinbis Facial Descriptors For Human Interation Recognition in Still Images, 2016, pp. 44-51.

[2] JifengShena, XinZuob, Jun Lic, WankouYangc, Haibin Ling A novel pixel neighborhood differential statistic feature for pedestrian and face detection. Pattern Recognition 63 (2017) 127-138.

[3] SaranuKavyaPooja, KogantiKoundinya, J Hemamalini Efficiency Measurement of Detecting Object From Video. International Journal of Applied Engineering Research, ISSN 0973-4562, Volume 10, Number 5 (2015) pp. 12165-12175.

[4] S. Zhang, C. Bauckhage, A.B. Cremers Informed Haar-Like Features Improve Pedestrian Detection. in: Proceedings of the IEEE Conference on Computer Vision and Pattern Recognition, 2014.

[5] B.Menser, F.Muller Face Detection In Color Images Using Principal Components Analysis. 1999, volume-2, pp. 620-624.

[6] Changjun Zhou, Lan Wang, Qiang Zhang, Xiaopeng Wei Face Recognition Based on PCA And Logistic Regression Analysis. 2014, pp. 5916-5919.

[7] J Hemamalini, D Kaavya Analysis and Efficiency of Error Free Compression Algorithm for Medical Image. Journal of Theoretical and Applied Information Technology, ISSN: 1992-8645, 20th April 2015. Vol.74 No.2, pp. 269-273

[8] J. Hemamalini and Merlin Pauliesther Survey of Recent Research and Issues in Video Surveillance. International Journal of Applied Engineering Research, ISSN: 0973-4562 Volume 9, Number 24 (2014) pp. 23477-23484.

[9] Liton Chandra Paul, Abdulla Al Sumam Face Recognition Using Principal Component Analysis Method. 2012, vol1, Issue-9, pp.135139.

[10] L.Bossard, M.Guillaumin, L.VanGool Event Recognition in Photo Collection with a Stopwatch HMM. in: Proceedings on the IEEE International Conference on Computer Vision, 2013, pp.1193-1200.

[11] M.A. Turk, A.P. Pentland Eigenfaces for recognition, J. Cogn. Neurosci., (1)(1991) 71-86.

[12] M. Kirby, L. Sirovich Application of the KL procedure for the characterization of human faces. IEEE Trans. Pattern Mach. Intell. 12 (1) (1990)103-108.

[13] Phillip Ian Wilson, Dr. John Fernandez, Facial Features Detection Using Haar Classifiers. pp.127-133

[14] Richard Jiang, Anthony T. S. Ho, Ismahane Cheheb, Noor Al-Maadeed, Somaya Al-Maadeed , AhemdBouridane, Emotion Recognition From Scrambled Facial Images Via Many Graph Embedding. 2017.

[15] S. Rajkumar and J. Hemamalini, RetriveSimilarty Facial And Disguise Image From Webcam Using Unsupervised Label Refinement Technique. Global Journal of Pure and Applied Mathematics. ISSN 0973-1768, Volume 11, Number 5 (2015), pp. 3713-3725.

[16] S. Zhang, R. Benenson, B. Schiele Filtered Channel Features for Pedestrian Detection in: Proceedings of the IEEE Conference on Computer Vision and Pattern Recognition, 2015, pp. 1751-1760.

[17] ShuzheWua ,Meina Kana, ZhenliangHea, Shiguang Shana, Xilin Chena Funnel-Structured Cascade for Multi-View Face Detection with Alignment Awareness. Neurocomputing 221 (2017) 138-145.।

[18] P.Viola,M.Jones, Rapid Object Detection Using a Boosted Cascade of Simple Features. 2001, pp.511-518.

\section{AUTHOR PROFILE}

Hemamalini $\mathbf{J}$ is presently Assistant Professor in the Department of Information Technology, SathyabamaUniversity, Chennai, India. She obtained her master degree from Sathyabama University, Chennai. She pursuing Ph.D in Video Surveillance Her interests include image processing and video processing.

Sri Dhanya currently doing Under Graduation in Department of Information Technology at Sathyabama University, Her research interests include image Processing, video processing, and Web Services.

Nikita Idiculla currently doing Under Graduation in Department of Information Technology at Sathyabama University, Her research interests include image Processing, video processing, and Web Services. 